\title{
NEW DEVELOPMENTS IN NIOBIUM TITANIUM SUPERCONDUCTORS
}

\author{
D. C. Larbalestier and P. J. Lee \\ Applied Superconductivity Center, University Of Wisconsin-Madison, Madison, WI 53706 USA
}

After a very active period of Niobium Titanium superconductor strand development in the mid 1980s, progress slowed as the SSC scaled up for production and emphasis moved towards guaranteeing, rather than enhancing performance. However, this period also produced several advances in the understanding of flux pinning in Niobium Titanium alloys and in the fabrication of composites containing artificial pinning centers (APC). This paper reviews some of this progress and attempts to assess its implications for future accelerator conductors.

\section{STATE OF THE ART}

\section{A. Overview of the development of properties with time}

The High Energy Physics (HEP) accelerator community has been the most consistent and effective driving force behind the major advances that useful superconductors have achieved in the last 30 years. The principal material advanced is Niobium Titanium of composition Nb-47wt.\% Ti. There are at least three major reasons why the HEP community has played such a large role in superconducting wire development: One is that the accelerator community knows that increased magnet performance is always driven by an ever increasing critical current density $\left(J_{c}\right)$, two the broader HEP community has stuck with superconductor development, even when there was not an immediate big accelerator project and three that there has been a commitment from key figures in the community to encourage productive links between the basic science of flux pinning in superconductors, the accelerator magnet community and the superconducting industry. In a series of workshops sponsored by the US Department of Energy, Division of High Energy Physics, held at least annually from 1983 until the present day, a community of US researchers, users and manufacturers have catalyzed each other in a continued attempt to wrest more performance from $\mathrm{Nb}$ - Ti conductors. Figure 1 collects information on some of the advances in performance of representative strands of this material. This progress is impressive. Figure 1 contains information on present and future applications of $\mathrm{Nb}$-Ti. The early large scale US procurements of conductors for Mirror Fusion Test Facility (MFTF) and for the Fermilab Tevatron in about 1980 developed the industry. Production of hundreds of billets generated genuine production statistics and also allowed researchers like ourselves to ask questions about which fabrication variables were really important in controlling the then rather variable, properties. The Fermilab $J_{c}(5 \mathrm{~T}, 4.2 \mathrm{~K})$

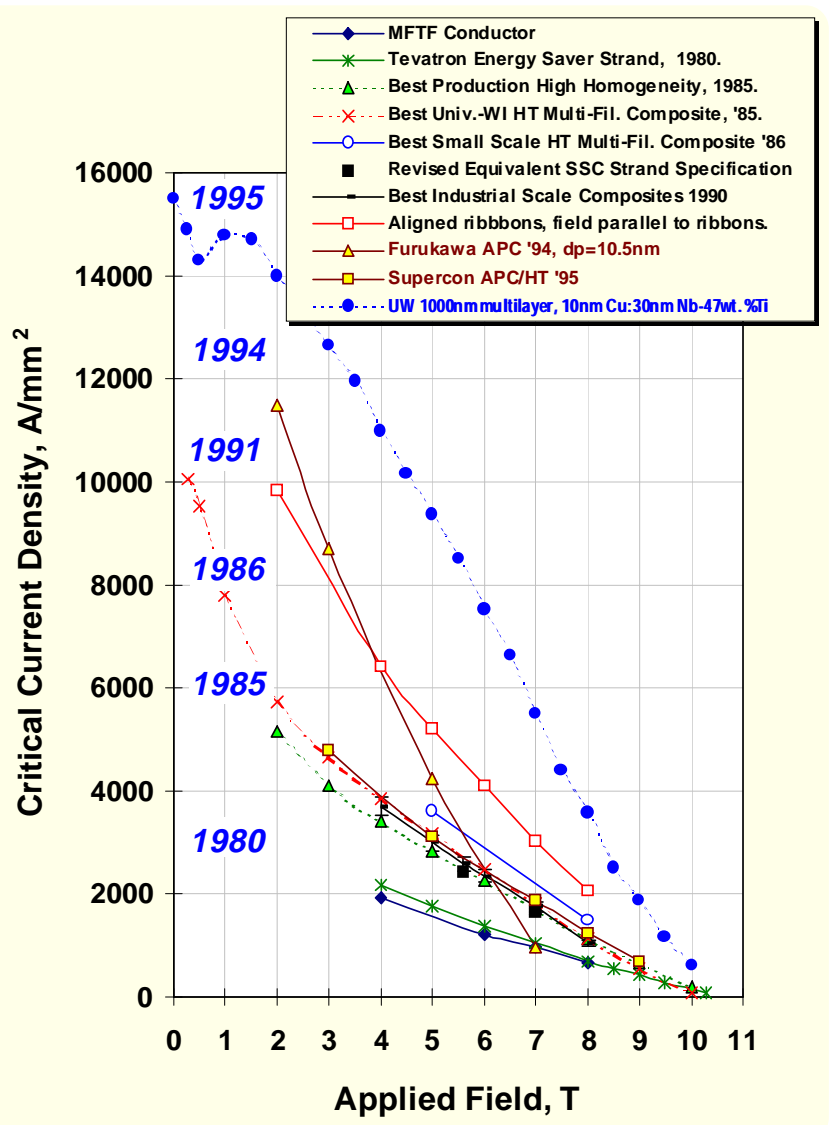

Figure 1 Advances in the Critical Current Density of $\mathrm{Nb}$-Ti Based Superconductors. All data are at 4.2K. Key: MultiFil = Multifilamentary strand, HT = Precipitation Heat Treated, APC = Artificial Pinning Center (aligned ribbons ref. [1], Furukawa APC ref. [2], Supercon APC/HT ref.[3]).

specification of $1800 \mathrm{~A} / \mathrm{mm}^{2}$ was less a carefully thought out attempt to determine what the material was capable of than an attempt to challenge production to a consistent, deliverable level. Its realization required simultaneous work on the raw alloy, the copper, the extrusion process, large rod drawing and fine wire drawing and the heat treatment capabilities of a still small industry. Without a firm idea of what the empirical optimization processes employed by industry really were doing to the microstructure and thus to the flux pinning which controls $J_{c}$, it is not surprising that there was eventually a considerable extra margin of performance to be found in the material, the exploitation of which in the mid to late 1980s led to the SSC benchmark $50 \%$ larger of about $2750 \mathrm{~A} / \mathrm{mm}^{2}$, while at the same time diminishing the filament size from $\sim 10$ to $6 \mu \mathrm{m}$. 
Underpinning the big jump from 1980 to 1985 was the discovery of the large role played by uncontrolled microscale chemical inhomogeneities in the raw $\mathrm{Nb}$-Ti alloy[4]. When, with a great deal of help from collaborations with R. M Scanlan (LBL) and W. K. McDonald at Teledyne Wah Chang Albany (TWCA) [5],the first high homogeneity Nb-Ti alloy was delivered, it became almost immediately possible to get the $50 \%$ increase in performance in an industrial scale billet. This improvement was based on a sound understanding of the factors controlling the microstructure $[6,7,8]$. Further exploitation of this understanding on a laboratory scale led to large filament conductors which could achieve $\sim 3700 \mathrm{~A} / \mathrm{mm}^{2}$, although with larger filaments[9]. Since 1985-1986, engineering the very best laboratory scale properties into very long length (several $\mathrm{km}$ ), very fine filament (6 $\mu \mathrm{m}$ or even lesser diameters) cabled conductors has been the principal emphasis of the industry. This was triumphantly done in the latter stages of the SSC-CDG R\&D program in a collaboration in which groups from the principal US industrial suppliers (IGC, OST, Supercon and TWCA) worked together with teams at principally BNL, Fermilab, LBL and the University of Wisconsin to characterize, understand and to devise engineering solutions that stood in the way of realizing the scientific control of microstructure that underpinned the industrial fabrication process (these results are reviewed in greater detail elsewhere[10]).

Two recent benchmarks significantly lift the potential of $\mathrm{Nb}$-Ti alloys. One was a proof-of principle experiment conducted in our group [1], aimed at answering the question of whether $\mathrm{Nb}$-Ti had absolutely run out of its capacity to develop any higher $J_{c}$. In this case we took an already very

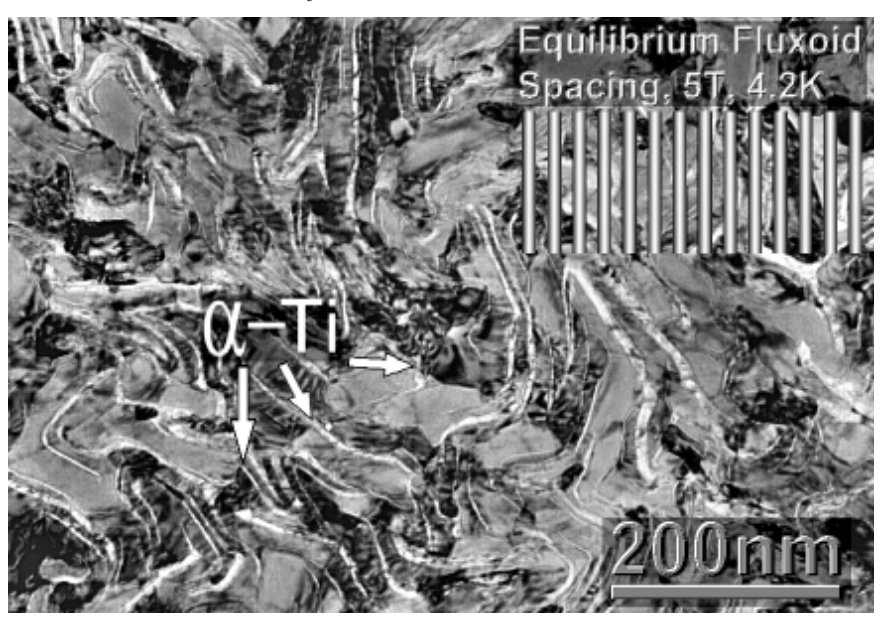

Figure 2 Typical Nb-47wt.\% Ti high critical current microstructure (in transverse cross-section) showing the densely folded sheets of $\alpha$-Ti pinning centers dispersed within the superconducting $\beta$-Nb-Ti matrix. Superimposed is a schematic illustration of the equilibrium fluxoid spacing and dimensions appropriate to $\mathrm{Nb}-47 \mathrm{wt} . \% \mathrm{Ti}$ at $5 \mathrm{~T}, 4.2 \mathrm{~K}$. high $J_{c}$ sample and rolled it to a high aspect ratio so that the pinning microstructure (Figure 2) was highly aligned for one axis of the wire. $J_{c}(5 \mathrm{~T})$ was raised from about 3500 to 5300 $\mathrm{A} / \mathrm{mm}^{2}$ without any degradation at higher fields. This proved that there was a greater potential in the material. The second aspect studied after 1985 was the question of whether a better array of pinning centers could be incorporated into $\mathrm{Nb}-\mathrm{Ti}$ than was supplied by nature, even in the highly optimized process that had evolved in the early 1980s. Artificial pinning center (APC) arrays were first introduced by Dorofejev et al. [14] at the 1985 Magnet Technology Conference, just as the conventional process was reaching its full understanding. The best realization of this concept is by Matsumoto et al. [2] from Furukawa Electric Co., as indicated by the 1994 plot in Figure 1. This composite was the first and is still the only round wire to have broken the $4000 \mathrm{~A} / \mathrm{mm}^{2}$ (5T) barrier. This result is all the more impressive in that the wire clearly lacks a competitive high field performance (it crosses over with standard wires at $\sim 6$ $\mathrm{T})$, as we discuss further later in this paper. In fact recent APC multilayers [11] and [12] have shown that highly aligned pinning layers can produce $>10,000 \mathrm{~A} / \mathrm{mm}^{2}$, first at a similar expense to the high field $J_{c}[11]$ and now without any compromise for the high field properties [12].

In the rest of this paper we briefly review some of the implications of these recent advances for new accelerator conductor possibilities, first briefly defining the difference between conventional and APC conductors.

\section{B. Conventionally Processed Material}

Virtually all material made up until now has been conventionally processed, where the term conventional means that the starting point is a $\mathrm{Nb}$ - Ti alloy which is sheathed in $\mathrm{Cu}$, and after various compaction, extrusion and drawing processes is then heat treated multiple times within the $\alpha+\beta$ two phase field so as to produce a fine dispersion of $\alpha$-Ti precipitates. These $\alpha$-Ti precipitates (Figure 2) are the dominant flux pinning centers in conventional wire and their optimization is the goal of the complex fabrication procedures through which $\mathrm{Nb}-\mathrm{Ti}$ composites are manufactured. Advanced accelerator strands optimized using such a process can today be expected to have critical current densities of the order of $2750-3000 \mathrm{~A} / \mathrm{mm}^{2}$ at $5 \mathrm{~T}, 4.2 \mathrm{~K}$ and $1100-1400 \mathrm{~A} / \mathrm{mm}^{2}$ at $8 \mathrm{~T}, 4.2 \mathrm{~K}$ and to consist of $1000-4000$

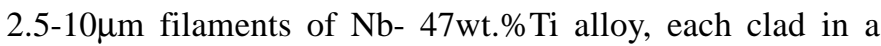
thin $\mathrm{Nb}$ diffusion barrier and assembled within a $\mathrm{Cu}$ matrix for stability (Figure 3). Inherent limitations of the conventional process are that $\alpha-\mathrm{Ti}$ is the pinning center and that the quantity of pinning center is limited to $20-25$ vol. $\%$ for $\mathrm{Nb}-47 \mathrm{Ti}$ by the thermodynamic phase stability of the $\mathrm{Nb}$ - Ti system. The shape of the pins is constrained to the ribbon-shaped pin morphology of Figure 2 by the plane strain 


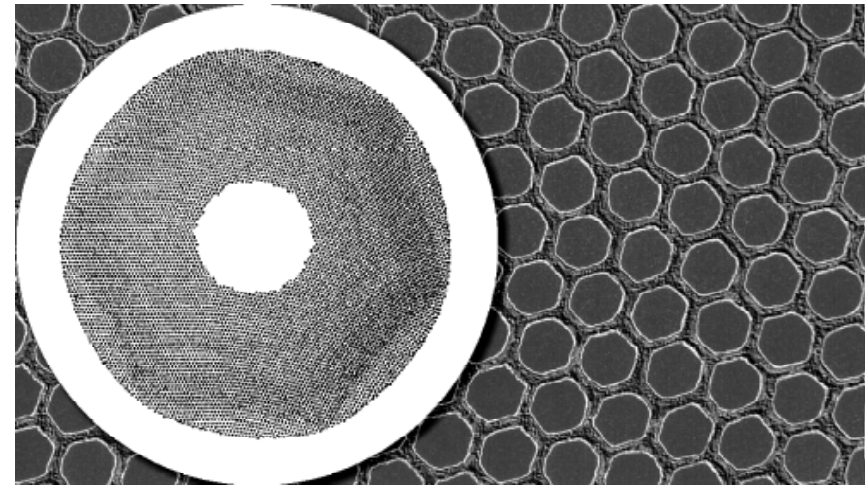

Figure 3 Cross-section of SSC-style multifilamentary superconducting strand at $26 \mathrm{~mm}$ diameter superimposed on detail showing filaments and their diffusion barriers (fabricated by IGC as part of the SSC-CDG R\&D program).

deformation dynamics of the body centered cubic $\beta-\mathrm{Nb}-\mathrm{Ti}$ alloy. It is an amazing gift from nature that it is possible to produce two phase nanometer scale microstructures such as that seen in Figure 2 in complex many thousand filament composites such as that shown in Figure 3 and to have the piece length be several $\mathrm{km}$. No wonder that competition with standard process $\mathrm{Nb}$-Ti is so hard!

\section{Artificial Pinning Centers (APC)}

An alternative approach, first used by Dorofejev et al. [14], is to mechanically assemble the desired pinning structure in a composite at large size and then to reduce the structure to the dimensions of the fluxoid lattice by multiple extrusions and wire drawing. Such a process has great conceptual flexibility in the selection of pin, superconducting matrix, and pinning center geometry. One realization of such a composite is shown in Figure 4. The figure shows an important and common attribute of APC composites, namely that the final size nanostructure is not the idealized macrostructure that was assembled. In fact the final size nanostructure of APC composites is seldom explicitly studied and this is certainly one of the key drawbacks to much recent work on trying to understand why disappointing performance

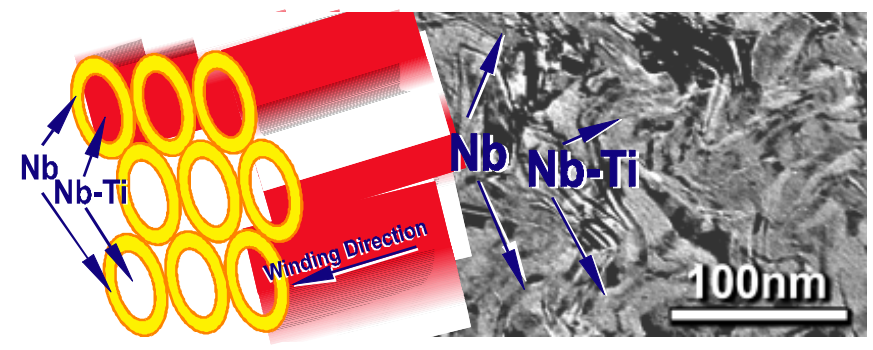

Figure 4 TEM image of original IGC APC at final size (in transverse cross-section) compared with the original "flux tube" design from the patent of Zeitlin et al. [13]. has so far been more common than outstanding performance. With these two concepts in mind we now turn to a wider discussion of the potential that the two processes offer for accelerator conductors.

\section{OUTSTANDING ISSUES FOR FUTURE DEVELOPMENT}

Three issues underlie the development of higher $J_{c}$ : The first is that of understanding the flux pinning mechanism, the second is ensuring that the upper critical field $H_{c 2}$ is not degraded by the choice of pinning center and the third is making composites that are fabricable in useful lengths at appropriate cost. We shall aim our discussion towards these 3 points.

\section{A. Flux-pinning.}

The keys to our present understanding of flux-pinning came from careful correlation of superconducting property measurements to a quantitative microstructural description. This turns out to be particularly necessary for understanding the APC composites, many of which do not have wellunderstood nanostructures [15]. The Volume Flux Pinning Force, $F_{p}\left(F_{p}=J_{c} \times B\right)$ is a particularly valuable optimization parameter. The microstructural and flux pinning basis of present optimization was described in the studies of Meingast, Lee and Larbalestier [16]. There are systematic changes in the shape of $F_{p}$ as the wire and its precipitates are drawn to different sizes, as is shown in Figure 5. Two important issues of great practical relevance are that drawing the wire to its optimum size raises $F_{p}$ at all fields and that the maximum in $F_{p}$ occurs at about $0.5 H_{c 2}$. This behavior is unlike that of APC Nb-Ti and almost all other high field superconductors and is the reason why $\mathrm{Nb}$ - $\mathrm{Ti}$ magnets are effective so close to their upper critical field. Two other very important results [17] are that $F_{p}$ and $J_{c}$ are linearly dependent on the volume percentage of pinning material and that the $H_{c 2}$ of the optimized composite is the same as that of the homogeneous starting alloy. This result was extremely surprising when first obtained because the matrix of the superconductor is strongly depleted of Ti by the formation of about 20 vol.\% of $\alpha$-Ti. This occurs because the optimum size precipitates (Figure 2) are thinner than the superconducting coherence length, $\xi$. Thus there is a remixing of matrix and pin so far as $T_{c}$ and $H_{c 2}$ are concerned. The enormously beneficial surprise of this result is that this remixing does not in any way lose the heterogeneous nanostructure required for optimum flux pinning. This principle has further important implications for the optimization of ternary $\mathrm{Nb}$-Ti-Ta compositions and APC composites. 
The question posed at the start of this section is whether flux pinning is well understood. At the conclusion of [16], the answer appeared to be a qualified yes. The microstructure and the flux pinning force had been directly correlated for the very highest $J_{c}$ composite over more than an order of magnitude change in pin dimension and $F_{p}$. By correlating these two it was possible to deduce the thickness dependence of the elementary pinning force and to compare this to calculations of the vortex core interaction with the pin. These calculations correctly predicted the observed linear dependence of the elementary pinning force $f_{p}$ on pin thickness up to a pin thickness of $2 \xi$, the vortex diameter. This was a considerable success. However, there was one flaw in the agreement.

The basis for flux pinning was long believed to be a core pinning interaction between the normal $\alpha$-Ti precipitate and the normal vortex core. However, the $\alpha$-Ti pins are so narrow that they are proximity coupled to the matrix, thus creating an important theoretical complication not treated in the simple models used to compute $f_{p}$. A second issue is that the thickness dependence of $f_{p}$ was found to fit best in the limit of T tending to $T_{c}$, where the field dependence of $F_{p}$ was not a good fit to the field dependence of the order parameter. This apparently small inconsistency was attacked by Cooley et al. [19] in the context of the shape of the $F_{p}(H)$ curves of APC conductors, virtually all of which exhibit a peak at low

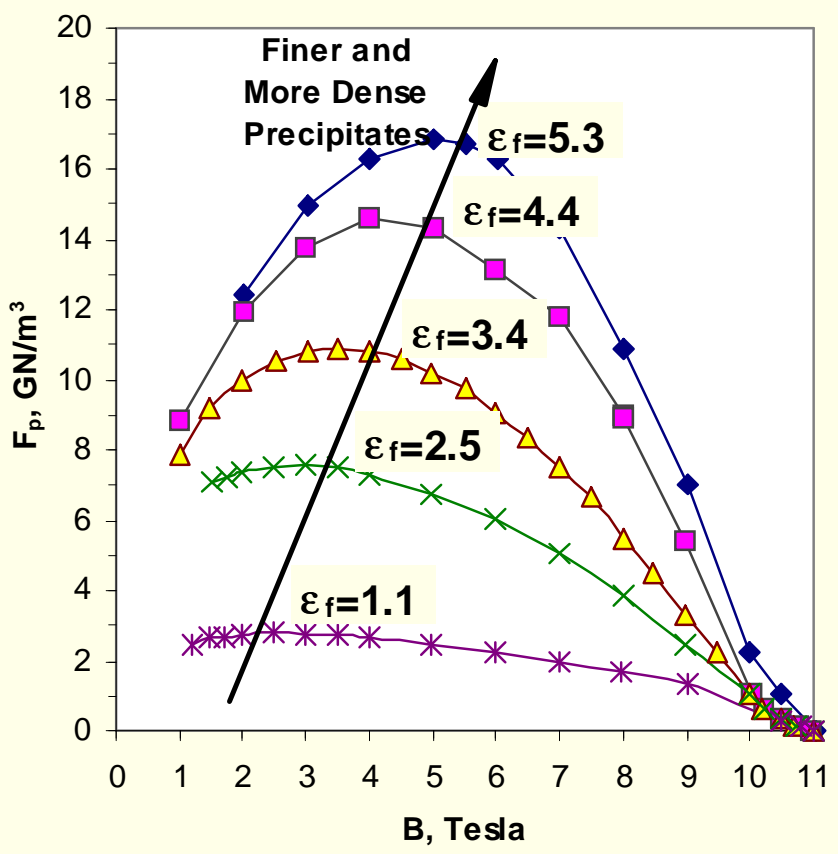

Figure 5 For conventionally processed $\mathrm{Nb}$-Ti the bulk pinning force increases in magnitude with drawing strain after the last heat treatment. The increase occurs at all fields as the precipitate size and spacing are reduced to less than a coherence length in thickness (see Figure 2) (data from [18]). fields, about $0.25 H_{c 2}$, rather than the $0.5 H_{c 2}$ of the conventional materials (see Figure 1 and Figure 5). The proposal was made there without much justification that the pinning mechanism in the APC was a magnetic one, rather than a core pinning one. In fact it now seems that this may be the dominant mechanism in conventional Nb-Ti too [20,21]. A key prediction of the new model [21] is that optimum $f_{p}$ occurs when the pin is decoupled from the matrix. The thickness of the pin and its electron mean free path determine this.

Within this model it is easy to rationalize the poor high field performance of most APC conductors: The reason is that their pins are pure $\mathrm{Nb}$, which has a much longer coherence length than the alloyed $\alpha$-Ti precipitate of the conventional process. The consequence of being optimized for thicker $(\sim 2-4 \xi)$ pins is that their density is lower than for thinner pins, thus reducing the number density of vortex-pin interactions. Thus the low field pinning benefits strongly but the higher field suffers. The remedy is thus to dirty up the pin. Experimental tests of this hypothesis are now underway.

This is not quite the whole story since Figure 1 shows that the $H_{c 2}$ of the very good APC wire is $\sim 7.5 \mathrm{~T}$, rather than the $10.5 \mathrm{~T}$ of conventional wires. This is principally the consequence of the billet being experimental. $\mathrm{Nb}-47 \mathrm{wt} . \% \mathrm{Ti}$ was used for the matrix and $\mathrm{Nb}$ for the pins, meaning that the overall composition was very far from the optimum $H_{c 2}$ composition. As the correct choice of pinning center becomes better understood this aspect of the reduced high field performance will be designed out by making sure that the overall composition of matrix and pin is one with a high $H_{c 2}$.

One final note about APC wires should be made. The most successful one practically has been the jellyroll design of Supercon that uses co-wound sheets of pure $\mathrm{Nb}$ and $\mathrm{Ti}$. This technique produces a strand that is claimed to be cost competitive with conventionally made material. Both dipole and MRI magnets have been made with the design. The properties are very similar to conventional $\mathrm{Nb}$-Ti but do not exceed the normal material. At this stage the nanostructure produced in the wires is not very clear.

\section{B. Upper Critical Field.}

A contour plot of $\mathrm{H}_{c 2}$ at $2 \mathrm{~K}$ for alloys in the ternary $\mathrm{Nb}$ Ti-Ta system is shown in Figure 6. Ta and $\mathrm{Nb}$ are mutually soluble, similar chemically and mechanically and $H_{c 2}$ $(\mathrm{T}<4.2 \mathrm{~K})$ is greater in the ternary than in the binary. As indicated by the figure, $H_{c 2}(2 \mathrm{~K})$ can be increased from a maximum of 14.25 to $15.5 \mathrm{~T}$. These data indicate the prime reason for using $\mathrm{Nb}$-Ti-Ta. In most other respects except one, the alloy processes well and can be used to replace the binary alloy when 1.8-2 $\mathrm{K}$ operation is required. So far at least 4 
fairly large billets have been made of different alloys in the system:
Nb- 43wt.\% Ti-25wt.\% Ta
1980 UW[22,23]
Nb- 41wt.\% Ti-15wt.\% Ta
1983 Fermi/UW[4]
Nb- 44wt.\% Ti-15wt.\% Ta
1987 Fermi/UW[24,25]
Nb-37wt.\% Ti-22wt.\% Ta

None of these has had the maximum $H_{c 2}$ composition, principally because of the desire to minimize the more expensive Ta.

Any new alloy must strike a balance between ensuring that three is enough Ta to raise $H_{c 2}$ and enough Ti to get sufficient $\alpha$-Ti for pinning. In fact the best $\mathrm{Nb}$-Ti-Ta alloys made so far have had $J_{c}(4.2 \mathrm{~K})$ values which are a little lower than the binary but they do improve at $2 \mathrm{~K}$. There have been few problems with $\mathrm{Nb}$-Ti-Ta alloy, making it a rather simple retrofit if $2 \mathrm{~K}$ use is being considered.

\section{Fabricability}

Conventional $\mathrm{Nb}-\mathrm{Ti}$ and $\mathrm{Nb}-\mathrm{Ti}-\mathrm{Ta}$ fabricate extremely well, often producing piece lengths exceeding several $\mathrm{km}$. Thus there is no concern about them. By contrast, APC conductors are sometimes quite hard to make. The problems are of several types. First is the fact that multiple extrusions

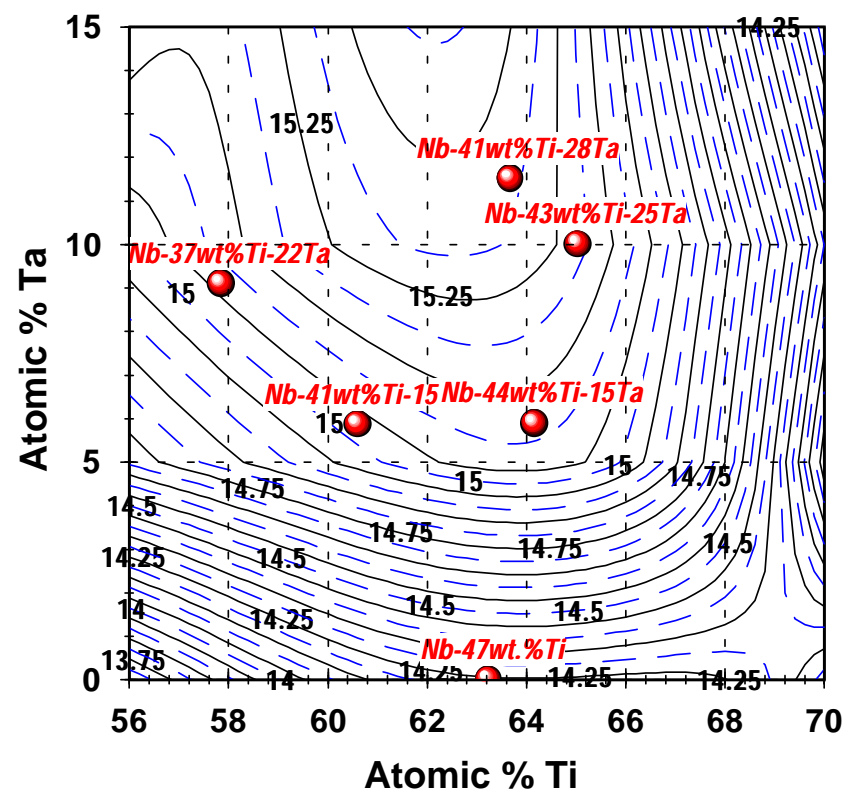

Figure 6 Contour plot of $H_{c 2}$ at $2 \mathrm{~K}$ for Nb-Ti-Ta from the data of Hawksworth [22] and Muller [27]. The investigated alloy compositions are superimposed on this plot along with the standard binary $\mathrm{Nb}-47 \mathrm{wt} . \% \mathrm{Ti}$. We use atomic composition here rather than the commonly used weight percentage because the all important volume $\%$ of a-Ti is more closely related to the atomic \% $\mathrm{Ti}$ and the weight percentage values for the ternary are often misleading in this respect.

are required to make an APC, except in the special case of the Supercon design mentioned above. Since each extrusion has a yield of $\sim 85 \%$ at maximum, it is easy to see that a design requiring 3 or 4 extrusions has an inevitably low yield. An additional problem is that the composite is twophase from the very beginning of fabrication and this leads to early work hardening and sometimes to fracture. This does not have to lead to problems but it is probably true that wire breaks are more common than with the standard process. These issues will take a greater importance as more of them made in an industrial environment.

\section{SUMMARY}

Recent advances in $\mathrm{Nb}$-Ti alloys have been reviewed. A better understanding of flux pinning in the system offers opportunities for new and better prototype designs, perhaps of multilayers and APC conductors. APC composites as presently made suffer from having the wrong overall composition and perhaps also from having pure metal pins. Ternary alloys should be capable of further optimization since too few having close to optimum compositions have yet been made. Near term designs of specialty magnets for low beta quadrupoles may provide a stringent test of the potential of these new concepts, since applications of this type are not too sensitive to conductor cost. It does appear that $\mathrm{Nb}-\mathrm{Ti}$ conductors with better properties can still be developed. The challenge is to integrate the lessons of the much better flux pinning properties of multilayers into more conventional round wire designs.

We gratefully acknowledge discussions with many present and former UW colleagues, particularly Lance Cooley, Robert Heussner, Paul Jablonski, Erik Kadyrov and Alex Gurevich. We are also greatly indebted to continued collaboration and encouragement from Ron Scanlan and $\mathrm{Al}$ McInturff (LBL), Dave Sutter (DOE) and colleagues in the superconductor industry, particularly Eric Gregory (IGC), Seung Hong (OST), and William McDonald (TWCHuntsville). Early help from Bruce Strauss, formerly of Fermilab, is also gratefully acknowledged.

\section{REFERENCES}

[1] L. D. Cooley, P. D. Jablonski, P. J. Lee and D. C. Larbalestier, "Strongly enhanced critical current density in Nb-47 wt.\% Ti having a highly aligned microstructure," Appl. Phys. Lett. 58, pp. 2984-2986, 1991.

[2] K. Matsumoto, H. Takewaki, Y. Tanaka O. Miura, K. Yamafuji, K. Funaki, M. Iwakuma, and T. Matsushita, "Enhanced $J_{c}$ properties in superconducting NbTi composites by introducing $\mathrm{Nb}$ artificial pins with a layered structure," Appl. Phys. Lett, vol. 64(1), pp.115-117, 1994.

[3] J. M. Seuntjens, M. Rudziak, C. Renaud, T. Wong 
and J. Wong, "Development Progress of the Supercon APC Process," presentation to the 1995 Low Temperature Superconductor Workshop, Boulder CO, March 1995.

[4] A. W. West, W. H. Warnes, D. L. Moffat and D. C. Larbalestier, "Compositional inhomogeneities in $\mathrm{Nb}-\mathrm{Ti}$ and its alloys," IEEE Trans. Mag., 19, pp.749-753, 1983.

[5] D. C. Larbalestier, A. W. West, W. Starch, W. Warnes, P. Lee, W. K. McDonald, P. O'Larey, K. Hemachalam, B. Zeitlin, R. Scanlan and C. Taylor, "High critical current densities in industrial scale composites made from high homogeneity Nb46.5T," IEEE Trans. Mag., 21, pp. 269-272, 1985.

[6] A. W. West and D. C. Larbalestier, "Microstructural Changes Produced in a Multifilamentary $\mathrm{Nb}$-Ti Composite by Cold Work and Heat Treatment," Met. Trans. A, 15, pp. 843-852, 1984.

[7] D. C. Larbalestier and A. W. West, "New perspectives on flux pinning in Niobium-Titanium composite superconductors," Acta Metallurgica, 32, pp. 1871-1881, 1984.

[8] P. J. Lee and D. C. Larbalestier, "Development of nanometer scale structures in composites of $\mathrm{Nb}$-Ti and their effect on the superconducting critical current density," Acta. Met., 35, pp. 2526-2536, 1987.

[9] Li Chengren and D. C. Larbalestier, "Development of high critical current densities in Niobium $46.5 \mathrm{wt} \%$ Titanium," Cryogenics, 27, pp. 171-177, 1987.

[10] P. J. Lee and D.C. Larbalestier, "An examination of the properties of SSC Phase II R\&D strands," IEEE Transactions on Applied Superconductivity, 3, pp. 833-841, 1993.

[11] J. D. McCambridge, N. D. Rizzo, X. S. Ling, J. Q. Wang, D. E. Prober, L. R. Motowidlo and B. A. Zeitlin, "Flux pinning in NbTi/Nb multilayers," IEEE Trans. Appl. Superconductivity, pp. 1697-1699, 1995.

[12] E. Kadyrov, A. Gurevich and D. C. Larbalestier, "Very high critical current densities in $\mathrm{Nb}-47 \% \mathrm{Ti}$ multilayers with a planar copper flux pinning microstructure," submitted for publication in Appl. Phys. Let., September 1995

[13] B. A. Zeitlin, M. S. Walker and L. R. Motowidlo, "Superconductors having controlled laminar pinning centers, and method of manufacturing same," US Patent 4,803,310, 1989.

[14] G. L. Dorofejev, E. Yu Klimenko, and S. V. Frolov, "Current carrying capacity of superconductors with artificial pinning centers," Proceedings of the 9th International Conference on Magnet Technology, Ed. by C. Marinucci and P. Weymuth (pub. Swiss Institute for Nuclear Research, Villigen, Switzerland), pp. 564-566, 1985.

[15] P. J. Lee, D. C. Larbalestier, and P. D. Jablonski, "Quantification of Pinning Center Thickness in Conventionally Processed and Powder Processed Artificial Pinning Center Microstructures," IEEE Trans. Appl. Superconductivity, 4, pp. 1701-1712, 1995.
[16] C. Meingast, P. J. Lee and D. C. Larbalestier, "Quantitative description of a high $J_{c} \mathrm{Nb}$-Ti superconductor during its final optimization strain: I. Microstructure, $T_{c}, H_{c 2}$ and resistivity," J. Appl. Phys., 66, pp. 5962-5970, 1989.

[17] P. J. Lee, J. C. McKinnell, and D. C. Larbalestier, "Restricted Novel Heat Treatments for Obtaining High $\mathrm{J}_{\mathrm{c}}$ in Nb-46.5wt\% Ti," Adv. Cryo. Eng., 36, 287-294 (1990).

[18] C. Meingast and D. C. Larbalestier, "Quantitative description of a very high critical current density $\mathrm{Nb}-\mathrm{Ti}$ superconductor during its final optimization strain: II. Flux pinning mechanisms," J. Appl. Phys., 66, pp.5971-5983, 1989.

[19] L. D. Cooley, P. J. Lee and D. C. Larbalestier, "Is Magnetic Pinning a Dominant Mechanism in Nb-Ti?," IEEE Trans . Mag., 27, 1096-1100, 1991.

[20] A. Gurevich and L.D. Cooley, "Anisotropic flux pinning in a network of planar defects," Phys. Rev. B, 50, pp. 13568-13576, 1994.

[21] L. D. Cooley, P. J. Lee and D. C. Larbalestier, "Evidence that magnetic pinning is the dominant pinning mechanism in $\mathrm{Nb}-\mathrm{Ti}$ superconductors and the effect that proximity coupling of the pins to the matrix has on the pinning force," submitted for publication in Phys. Rev. B, August 1995.

[22] D.G. Hawksworth, and D.C. Larbalestier "Further investigations of the upper critical field and the high field critical current density in $\mathrm{Nb}-\mathrm{Ti}$ and its alloys," IEEE Trans. Mag., 17, pp. 49-52, 1981.

[23] H.R. Segal, T.M. Hrycaj, Z.J.J. Stekly, Z.J.J., T.A. de Winter, and K. Hemachalam, "Nb-Ti based conductors for use in 12 Tesla toroidal field coils," in Proc. of 8th Symp. on Engineering Problems in Fusion Research, IEEE Pub. 79CH-1441-5 NPS, pp.255-259, 1979.

[24] A. D. McInturff, Carson, D. C. Larbalestier, P. J. Lee, J. C. McKinnell, Kanithi, McDonald, O'Larey "Ternary Superconductor "NbTiTa" for High Field Superfluid Magnets," IEEE Inter. Magnet Conf., Digest of Technical Papers, Brighton Polytechnic, Brighton, U.K., p: AP15, 1990.

[25] P. J. Lee, D. C. Larbalestier, J. C. McKinnell and A. D. McInturff, "Microstructure property relationships in Nb-Ti-Ta," IEEE Transactions on Applied Superconductivity, 3, pp. 1354-1357, 1993.

[26] B. G. Lazarev, O. V. Chernyj, G. E. Storozhilov, L. G. Udov, N. F. Andrievskaya, L. A. Kornienko, L. S. Lazareva, N. A. Chernyak, P. A. Kutsenko, B. K. Pryadkin, Y. A. D. Starodubov, M. B. Lazareva and V. M. Gorbatenko, "The Study of the Microstructure and $J_{c}$ in Nb-37Ti-22Ta Superconductor Produced with Different Duration of Treatments," Proc. 7th Int. Workshop on Critical Currents in Superconductors, Alpbach, Austria, Ed. H. W. Weber, World Scientific Press, Singapore, 1994.

[27] H. Muller, "The upper critical field of niobiumtitanium," Ph.D. Thesis, University of Wisconsin-Madison, 1988. 